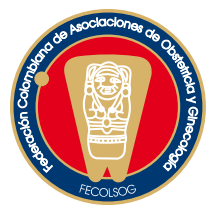

\title{
ENDOMETRIOMA E INFERTILIDAD: REVISIÓN DE ALTERNATIVAS TERAPÉUTICAS
}

\section{Endometriomas and infertility: a review of therapeutic alternatives}

Gustavo Adolfo Restrepo-Cano, M.D.*

Recibido: junio 2/10 - Aceptado: enero 17/11

\section{RESUMEN}

Objetivo: realizar una revisión de la literatura sobre el tratamiento de los endometriomas en el área de la infertilidad.

Materiales y métodos: el presente trabajo es una revisión sistemática cualitativa, para la cual se realizó una búsqueda electrónica en PubMed/ MEDLINE, Hinari y Cochrane, para recopilar información publicada tanto en inglés como en español desde febrero del 2000 a marzo del 2010, eligiendo aquellos artículos que evaluaban el tratamiento de los endometriomas en función de la infertilidad.

Resultados: la mejor evidencia respecto al manejo de los endometriomas en infertilidad se encuentra en las diferencias de las técnicas quirúrgicas para su tratamiento. La cistectomía laparoscópica es el método quirúrgico más efectivo, afecta de menor manera la reserva ovárica folicular y disminuye de forma significativa el riesgo de recurrencias. No afecta los resultados de la FIV (fertilización in vitro) en términos de embarazos.

Conclusiones: la cirugía excisional laparoscópica (cistectomía) de los endometriomas ofrece ventajas frente a las demás.

Palabras clave: endometrioma, infertilidad, endometriosis, cistectomía, laparoscopia.

* Médico Gineco-obstetra, Universidad del Quindío. Residente de III año de Gineco-obstetricia, Universidad Militar Nueva Granada. Bogotá (Colombia).Correo electrónico: Medicgus9@hotmail.com.

\section{SUMMARY}

Objective: reviewing the pertinent literature concerning endometrioma treatment in the area of infertility.

Materials and methods: this work consisted of a qualitative systematic review involving a search of the PubMed/MEDLINE, Hinari and Cochrane databases to compile information published in both English and Spanish from February 2000 to March 2010, choosing those articles which evaluated treatment of endometriomas regarding infertility. Results: the best available evidence regarding the management of endometriomas in infertility was found in the differences between surgical techniques for treating them. Laparoscopic cystectomy was seen to be the most effective surgical method; it had the least effect on ovarian follicular reserve and significantly reduced the risk of recurrences. It did not affect in vitro fertilization (IVF) results in terms of pregnancies.

Conclusions: laparoscopic excision surgery (cystectomy) of the endometriomas offers advantages when compared to other available techniques.

Key words: endometrioma, infertility, endometriosis, cystectomy, laparoscopy.

\section{INTRODUCCIÓN}

La endometriosis se define como la presencia de glándulas endometriales y estroma fuera de la cavidad endometrial y de la musculatura uterina. ${ }^{1}$ Esta es responsable hasta del 20\% de la infertilidad 
de la pareja. ${ }^{2}$ La endometriosis del ovario representa el 52\% de las zonas de afectación; en dicho órgano, la endometriosis puede presentarse en forma de implantes superficiales o como masas pélvicas compuestas quísticas y sólidas (endometriomas) y más del 90\% de estos son pseudoquistes formados por invaginación de la corteza ovárica, tras lo cual reemplazan el tejido normal. ${ }^{3}$

La patogénesis de los endometriomas no está clara. Una hipótesis es el paso retrógrado de la sangre menstrual o el derramamiento de depósito de los implantes de endometriosis en el ovario. La invaginación progresiva de la corteza ovárica con estos depósitos lleva a la formación de un endometrioma, que es en realidad un pseudoquiste. ${ }^{4}$ Una vez que se desarrolla el quiste endometriósico ovárico, el inhibidor del activador del plasminógeno tipo 1 (PAI-1) y el inhibidor de la matriz de metaloproteinasa tipo 1 (TIMP-1) aumentan, lo cual puede explicar cómo frecuentemente se encuentran endometriomas sin invasión del tejido ovárico adyacente, el PA y la MMP3 se han descrito como moléculas favorecedoras del desarrollo de la endometriosis peritoneal. ${ }^{5}$

Con respecto al diagnóstico, este se basa en la histopatología. ${ }^{6}$ Igualmente, la ecografía es útil para apoyar el diagnóstico clínico de endometrioma y su presencia sugiere una endometriosis moderada a grave. ${ }^{7}$ Tiene una sensibilidad del $83 \%$ y una especificidad del $89 \%,{ }^{8}$ aunque el ultrasonido transvaginal es más sensible que el transabdominal para la detección de los endometriomas. ${ }^{9}$ También se han utilizado los marcadores tumorales como el CA 125 parta el diagnóstico de endometriosis en estadio III o IV. Niveles mayores de $100 \mathrm{UI} / \mathrm{ml}$ (normal $<35 \mathrm{IU} / \mathrm{ml}$ ) se asocian principalmente con enfermedad intraperitoneal extensa, adherencias o rotura de un endometrioma. ${ }^{10}$

Algunas veces, los endometriomas tienen un componente nodular sólido y sumado a la frecuentemente encontrada pared gruesa, lo que hace que sea difícil distinguirlos de una neoplasia. ${ }^{11}$ La presencia de fibras nerviosas en lesiones endometriósicas ováricas se ha comprobado mediante inmunotécnicas (PGP9.5). Esto puede explicar una vía en la fisiopatología del dolor en mujeres con endometriomas. ${ }^{12}$

En la última década, se ha publicado una importante cantidad de información que se ha generado en torno a los endometriomas y sus repercusiones en la fertilidad. ${ }^{13}$ Los mecanismos postulados que conducen a la alteración de la fertilidad van desde la distorsión anatómica de la pelvis hasta la producción de sustancias que son hostiles a la función normal del ovario. ${ }^{14} \mathrm{Si}$ la resección de estos conduce a mejorar la fertilidad, es un tema aún controvertido. ${ }^{15}$ Se pretende, con el artículo, hacer una aproximación sobre el tratamiento y técnica quirúrgica que aporta los mejores beneficios en cuanto al aumento de las tasas de embarazo y/o a la mejoría de los resultados de las técnicas de reproducción asistida en mujeres con endometriomas.

\section{MATERIALES Y MÉTODOS}

Se realizó una búsqueda en el Registro Central Cochrane de Ensayos Controlados y su grupo de Trastornos Menstruales y Subfertilidad, así como en MEDLINE, HINARI, PubMed y EMBASE, usando los términos MESH "endometriosis", "endometrioma”, "infertilidad", "cistectomía" y "laparoscopia". Finalmente, se eligieron ensayos controlados aleatorizados, revisiones cuantitativas y estudios retrospectivos desde febrero del 2000 hasta marzo del 2010 que compararon y/o evaluaron los diferentes tratamientos de los endometriomas y su repercusión en la infertilidad femenina.

\section{RESULTADOS}

Se obtuvieron 270 referencias. Posteriormente, se eligieron 60 artículos que evaluaron el tratamiento de los endometriomas en función de la infertilidad y que cumplieron con el objetivo planteado. A continuación, se presenta la evidencia encontrada.

\section{Infertilidad y endometrioma}

Continúa siendo polémico si la resección de un endometrioma asintomático mejora la fertilidad. 
Una preocupación importante es que la resección de los endometriomas resulta en la pérdida de pequeños folículos adyacentes a la pared del quiste, lo que lleva a que exista solo un grupo reducido de ovocitos y que la fertilidad se vea comprometida. ${ }^{16}$ Esta posibilidad ha sido apoyada por varios estudios que compararon el ovario operado con el ovario contralateral durante la hiperestimulación para la fertilización in vitro (FIV) y se observó que el ovario operado produjo menos folículos dominantes, ovocitos y embriones de alta calidad que el ovario intacto. ${ }^{17-19}$ Pero estas alteraciones no impactan en las tasas de embarazo. ${ }^{20,21}$ Incluso en una serie de casos, 3 de 126 pacientes desarrollaron insuficiencia ovárica inmediatamente después de la extirpación de los endometriomas ováricos bilaterales. ${ }^{22}$ Un interesante estudio clínico, en el cual se observó, mediante seguimiento folicular, a 71 pacientes con endometriomas unilaterales que no habían sido operadas, encontró tasas de ovulación del 22\%, lo cual resulta importante dado que la tasa esperada en ovarios normales es del $50 \% .^{23}$

La cistectomía laparoscópica de los endometriomas $>4 \mathrm{~cm}$ de diámetro mejora la fecundidad en comparación con el drenaje y la coagulación. ${ }^{7}$ Se recomienda la cistectomía laparoscópica para lesiones mayores o iguales a $4 \mathrm{~cm}$ de diámetro para confirmar el diagnóstico histológico, mejorar el acceso a los folículos, disminuir el riesgo de infección y posiblemente mejorar la respuesta ovárica. ${ }^{24}$

Respecto a los endometriomas y la fertilización in vitro, se ha descrito que la presencia de un endometrioma requiere dosis superiores de gonadotropinas. Asimismo, una estimulación más larga lleva a una reducción ovocitaria, pero no impacta en el resultado de la FIV en términos de embarazo. ${ }^{25-29}$ Demirol y Khamsi argumentan en favor de la no intervención dado que la contaminación de los ovocitos durante la punción por el líquido endometriósico afecta la capacidad para formar el embrión o la calidad del mismo. ${ }^{30,31}$ Pabuccu solo la recomienda para aquellos mayores a $6 \mathrm{~cm} .^{32}$
Por su parte, García-Velasco y Wong encontraron diferencias mínimas en los resultados de la FIV después de la primera o segunda intervención de los endometriomas, en términos del número de oocitos recogidos y tasas de embarazo. ${ }^{33,34}$

En cuanto a la técnica quirúrgica, el retiro de la pared del quiste en lugar de la realización de una incisión circular, seguida de la extracción, parece reducir el número de folículos normales eliminados con el espécimen patológico. ${ }^{35} \mathrm{El}$ análisis histológico de endometriomas demostró que la endometriosis de la pared del quiste rara vez penetra más de $1,5 \mathrm{~mm} .{ }^{36}$ Por lo tanto, la atención a la técnica parece ser crucial para la preservación de la función ovárica. ${ }^{37}$

En muchas mujeres, los endometriomas coexisten con quistes hemorrágicos, de cuerpo lúteo, o quistes foliculares. Estos quistes adyacentes a menudo aumentan la dificultad en la resección del endometrioma y deben ser dejados in situ cuando sea posible, en un esfuerzo por retener la mayor cantidad de tejido ovárico normal. La cistectomía (extirpación del quiste completo) realizada por laparoscopia es el enfoque terapéutico recomendado sin embargo no hay estudios controlados y aleatorizados que evalúen la cistectomía por laparotomía. ${ }^{38} \mathrm{La}$ aspiración es ineficaz, y se asocia con una tasa de recurrencia del 88 por ciento a los seis meses de seguimiento. ${ }^{39,40}$ La fenestración y la ablación (es decir, la eliminación de parte de la pared del quiste seguida de la coagulación o vaporización con láser de la parte interna de la pared) es también menos eficaz que la escisión, tanto en términos de mejoramiento de la fertilidad como de reducción del dolor. Estas conclusiones están sustentadas por los datos agrupados de un ensayo aleatorizado, un cuasi experimento, y dos estudios observacionales. ${ }^{41}$ En una revisión de Cochrane, que además incluye dos estudios aleatorizados sobre tratamiento laparoscópico de los endometriomas ováricos de más de $3 \mathrm{~cm}$ de tamaño, se concluyó que en comparación con el drenaje y la electrocoagulación del quiste, la escisión 
laparoscópica de la pared del quiste se asoció con una reducción de la recurrencia de los endometriomas (OR 0,41, IC 95\% 0,18-0,93), menor necesidad de cirugía posterior (OR 0,21; IC 95\% 0,05-0,79), reducción de las tasas de recurrencia de los síntomas de dismenorrea (OR 0,15, IC 95\% 0,06-0,38), dispareunia (OR 0,08, IC 95\% 0,01-0,51) y dolor pélvico no menstrual (OR: 0,10; IC 95\% 0,02-0,56), también se asocia con un aumento de la tasa de embarazo en mujeres cuya subfertilidad se había documentado (OR 5,21, IC 95\% 2,04-13,29). ${ }^{38}$

Tres series con un total de más de 1.200 pacientes informaron sobre las tasas de nueva formación de endometriomas y estas fueron al menos del 30\% de dos a cinco años después de la escisión. Las mujeres más jóvenes, con endometriomas de mayor tamaño, con enfermedad grave y con una historia de tratamiento médico para la endometriosis se encuentran en mayor riesgo de recurrencia. ${ }^{42,43}$ Por el contrario, el embarazo y la lactancia parecen reducir el riesgo de recurrencia. ${ }^{44}$

En un ensayo clínico aleatorizado en el que un grupo recibió dosis bajas cíclicas de anticonceptivos combinados y el otro ningún tratamiento durante seis meses, se encontró que ambos grupos tuvieron un riesgo similar de recurrencia a los 24 y 36 meses. $^{45}$ Otros estudios retrospectivos han demostrado lo contrario, ofrecieron bajas dosis cíclicas de estrógeno-progestina monofásicos y encontraron menores tasas acumuladas de recurrencia a los 36 meses que las no usuarias. ${ }^{46,47}$

La eliminación excesiva de tejido ovárico normal adyacente al endometrioma es otra causa del agotamiento folicular. El daño ovárico severo, entendido como ausencia de crecimiento folicular tras la estimulación, es de aproximadamente el $13 \%$ en pacientes que son llevadas a resección de endometriomas. ${ }^{48}$ Las mujeres deben ser aconsejadas sobre los riesgos respecto a la reducción de la función ovárica después de la cirugía y la posible pérdida del ovario. Además, la decisión debe ser reconsiderada si ya se ha realizado cirugía ovárica previamente. ${ }^{28}$ No se recomienda la punción de los quistes endometriósicos ya que con esta se ha descrito un mayor riesgo de infección. ${ }^{49}$

Por último, después de una cistectomía la tasa de recurrencia del endometrioma es de aproximadamente 15 a 30 por ciento en los próximos cinco años. No hay evidencia de que a largo plazo la supresión médica con anticonceptivos cíclicos o continuos después de la cirugía pueda prevenir la recurrencia. ${ }^{50,51}$

\section{CONCLUSIONES}

El abordaje quirúrgico excisional por laparoscopia ha demostrado las menores tasas de recurrencia, de disminución de la reserva ovárica y otras complicaciones asociadas. La cistectomía laparoscópica de los endometriomas $>4 \mathrm{~cm}$ de diámetro mejora la fecundidad en comparación con el drenaje y la coagulación. La presencia de un endometrioma no impacta los resultados de la FIV en términos de tasas de embarazo.

\section{AGRADECIMIENTOS}

Al Dr. Vicente Carmona Pertuz, Coordinador del Departamento de Gineco-obstetricia del Hospital Militar Nueva Granada.

Igualmente, a los Doctores Ivonne Jeannette Díaz Yamal, Luis Ernesto Pérez Agudelo y Mike Jimmy Castañeda Castañeda por su contribución con el servicio de endocrinología ginecológica del Hospital Militar Central.

\section{REFERENCIAS}

1. Olive DL, Schwartz LB. Endometriosis. N Engl J Med 1993;328:1759-69.

2. Smith S, Pfeifer AM, Collins JA. Diagnosis and management of female infertility. JAMA 2003;290:1767-70.

3. Woodward PJ, Sohaey R, Mezzetti TP Jr. Endometriosis: radiologic-pathologic correlation. Radiographics 2001;21:193-216.

4. Hemmings R, Bissonnette F, Bouzayen R. Results of laparoscopic treatments of ovarian endometriomas: laparoscopic ovarian fenestration and coagulation. Fertil Steril 1998;70:527-9. 
5. Loh FH, Tan AT, Kumar J, Ng SC. Ovarian response after laparoscopic ovarian cystectomy for endometriotic cysts in 132 monitored cycles. Fertil Steril 1999; 72:316-21.

6. Donnez J, Wyns C, Nisolle M. Does ovarian surgery for endometriomas impair the ovarian response to gonadotropin? Fertil Steril 2001;76:662-5.

7. Beretta P, Franchi M, Ghezzi F. Randomized clinical trial of two laparoscopic treatments of endometriomas: cystectomy versus drainage and coagulation. Fertil Steril 1998;70:1176-80.

8. Guerriero S, Mais V, Ajossa S, Paoletti AM, Angiolucci M, Labate F, et al. The role of endovaginal ultrasound in differentiating endometriomas from other ovarian cysts. Clin exp obstet gynecol 1995;22:20-2.

9. Bhatt S, Kocakoc E, Dogra VS. Endometriosis: Sonographic spectrum. Ultrasound Q 2006;22:273-80.

10. Seracchioli R, Mabrouk M, Frascà C, Manuzzi L, Montanari G, Keramyda A, et al. Long-term cyclic and continuous oral contraceptive therapy and endometrioma recurrence: a randomized controlled trial. Fertil Steril 2008;93:52-6.

11. Patel MD, Feldstein VA, Chen DC, Lipson SD, Filly RA. Endometriomas: diagnostic performance of US. Radiology 1999;2 10:739-45.

12. Abbott JA, Hawe J, Clayton RD, Garry R. The effects and effectiveness of laparoscopic excision of endometriosis: a prospective study with 2-5 year follow-up. Hum Reprod 2003;18:1922-7.

13. Tsoumpou I, Kyrgiou M, Gelbaya TA, Nardo, LG. The effect of surgical treatment for endometrioma: a systematic review and meta-analysis. Fertil Steril 2009;92:75-87.

14. Cahill DJ. What is the optimal medical management of infertility and minor endometriosis?: Analysis and future prospects. Hum Reprod 2002;17:1135-40.

15. Ragni G, Somigliana E, Benedetti F, Paffoni A, Vegetti W, Restelli L, et al. Damage to ovarian reserve associated with laparoscopic excision of endometriomas: a quantitative rather than a qualitative injury. Am J Obstet Gynecol 2005;193:1908-14.

16. Exacoustos C, Zupi E, Amadio A, Szabolcs B, De Vivo B, Marconi D, et al. Laparoscopic removal of endometriomas: sonographic evaluation of residual functioning ovarian tissue. Am J Obstet Gynecol 2004;191:68-72.

17. Loh FH, Tan AT, Kumar J, Ng SC. Ovarian response after laparoscopic ovarian cystectomy for endometriotic cysts in 132 monitored cycles. Fertil Steril 1999;72:316-21.
18. Somigliana E, Ragni G, Benedetti F, Borroni R, Vegetti W, Crosignani PG. Does laparoscopic excision of endometriotic ovarian cysts significantly affect ovarian reserve? Insights from IVF cycles. Hum Reprod 2003;18:2450-3.

19. Nargund G, Cheng WC, Parsons J. The impact of ovarian cystectomy on ovarian response to stimulation during in-vitro fertilization cycles. Hum Reprod 1996;11:81-3.

20. Tsoumpou I, Kyrgiou M, Gelbaya TA, Nardo LG. The effect of surgical treatment for endometrioma on in vitro fertilization outcomes: a systematic review and meta-analysis. Fertil Steril 2009; 92:75-87.

21. Demirol A, Guven S, Baykal C, Gurgan T. Effect of endometrioma cystectomy on IVF outcome: a prospective randomized study. Reprod Biomed Online 2006;12:639-43.

22. Ho HY, Lee RK, Hwu YM, Lin MH, Su JT, Tsai YC. Poor response of ovaries with endometrioma previously treated with cystectomy to controlled ovarian hyperstimulation. J Assist Reprod Genet 2002;19:507-11.

23. Benaglia l, Somigliana E, Vercellini P, Abbiati A, Ragni G, Fedele L. Endometriotic ovarian cysts negatively affect the rate of spontaneous ovulation. Hum Reprod 2009;24:2183-6.

24. Kennedy S, Bergguist A, Charpon C. ESHRE guideline for the diagnosis and treatment of endometriosis. Hum Reprod 2005;20:2698-704.

25. Isaacs JD Jr, Hines RS, Sopelak VM, Cowan BD. Ovarian endometriomas do not adversely affect pregnancy success following treatment with in vitro fertilization. J Assist Reprod Genet 1997;14:551-3.

26. Somigliana E, Infantino M, Benedetti F, Arnoldi M, Calanna G, Ragni G. The presence of ovarian endometriomas is associated with a reduced responsiveness to gonadotropins. Fertil Steril 2006;86:192-6.

27. Suzuki T, Izumi S, Matsubayashi H, Awaji H, Yoshikata $\mathrm{K}$, Makino T. Impact of ovarian endometrioma on oocytes and pregnancy outcome in in vitro fertilization. Fertil Steril 2005;83:908-13.

28. Tinkanen $\mathrm{H}$, Kujansuu E. In vitro fertilization in patients with ovarian endometriomas. Acta Obstet Gynecol Scand 2000;79:119-22.

29. Yanushpolsky EH, Best CL, Jackson KV, Clarke RN, Barbieri RL, Hornstein MD. Effects of endometriomas on ooccyte quality, embryo quality, and pregnancy rates in in vitro fertilization cycles: a prospective, case-controlled study. J Assist Reprod Genet 1998;15:193-7. 
30. Demirol A, Guven S, Baykal C, Gurgan T. Effect of endometrioma cystectomy on IVF outcome: a prospective randomized study. Reprod Biomed Online 2006;12:639-43.

31. Khamsi F, Yavas Y, Lacanna IC, Roberge S, Endman M, Wong JC. Exposure of human oocytes to endometrioma fluid does not alter fertilization or early embryo development. J Assist Reprod Genet 2001;18:106-9.

32. Pabuccu R, Onalan G, Goktolga U, Kucuk T, Orhon E, Ceyhan T. Aspiration of ovarian endometriomas before intracytoplasmic sperm injection. Fertil Steril 2004;82:705-11.

33. Garcia-Velasco JA, Mahutte NG, Corona J, Zuniga V, Giles J, Arici A, et al. Removal of endometriomas before in vitro fertilization does not improve fertility outcomes: a matched, case-control study. Fertil Steril 2004;81:1194-7.

34. Wong BC, Gillman NC, Oehninger S, Gibbons WE, Stadtmauer LA. Results of in vitro fertilization in patients with endometriomas: is surgical removal beneficial? Am J Obstet Gynecol 2004;191:597-606.

35. Muzii L, Bellati F, Palaia I, Bellati F, Plotti F, Manci N, Zullo MA, et al. Laparoscopic stripping of endometriomas: a randomized trial on different surgical techniques. Part I: clinical results. Hum Reprod 2005;20:1981-6.

36. Muzii L, Bianchi A, Cristi E, Pernice M, Zullo MA, et al. Histologic analysis of endometriomas: what the surgeon needs to know. Fertil Steril 2007;87:362-6.

37. Reich H, Abrao MS. Post-surgical ovarian failure after laparoscopic excision of bilateral endometriomas: is this rare problem preventable?. Am J Obstet Gynecol 2006;195:339-40.

38. Hart R, Hickey M, Maouris P, Buckett W, Garry R. Excisional surgery versus ablative surgery for ovarian endometriomata: a Cochrane Review. Hum Reprod 2005;20:3000-7.

39. Vercellini P, Vendola N, Bocciolone L, Colombo A, Rognoni MT, Bolis G. Laparoscopic aspiration of ovarian endometriomas. Effect with postoperative gonadotropin releasing hormone agonist treatment. J Reprod Med 1992;37:577-80.

40. Saleh A, Tulandi T. Surgical management of ovarian endometrioma. Infertil Reprod Med Clin North Am 2000;11:61.
41. Vercellini P, Chapron C, De Giorgi O, Consonni D, Frontino G, Crosignani PG. Coagulation or excision of ovarian endometriomas?. Am J Obstet Gynecol 2003;188:606-10.

42. Kikuchi I, Takeuchi H, Kitade M, Shimanuki $\mathrm{H}$, Kumakiri J, Kinoshita K. Recurrence rate of endometriomas following a laparoscopic cystectomy. Acta Obstet Gynecol Scand 2006;85:1120-4.

43. Liu X, Yuan L, Shen F, Zhu Z, Jiang H, Guo SW. Patterns of and risk factors for recurrence in women with ovarian endometriomas. Obstet Gynecol 2007;109:1411-20.

44. Koga K, Takemura Y, Osuga Y, Yoshino O, Hirota Y, Hirata $\mathrm{T}$, et al. Recurrence of ovarian endometrioma after laparoscopic excision. Hum Reprod 2006;21:2171-4.

45. Muzii L, Marana R, Caruana P, Catalano GF, Margutti F, Panici PB. Postoperative administration of monophasic combined oral contraceptives after laparoscopic treatment of ovarian endometriomas: a prospective, randomized trial. Am J Obstet Gynecol 2000;183:588-92.

46. Vercellini P, Somigliana E, Daguati R, Vigano P, Meroni F, Crosignani PG. Postoperative oral contraceptive exposure and risk of endometrioma recurrence. Am J Obstet Gynecol 2008;198:504.e1-5.

47. Seracchioli R, Mabrouk M, Manuzzi L, Vicenzi C, Frascà C, Elmakky A, et al. Post-operative use of oral contraceptive pills for prevention of anatomical relapse or symptom-recurrence after conservative surgery for endometriosis. Hum Reprod 2009;24:2729-35.

48. Benaglia L, Somigliana E, Vighi V, Ragni G, Vercellini P, Fedele L. Rate of severe ovarian damage following surgery for endometriomas. Hum Reprod 2010;25:678-82.

49. Kubota T, Ishi K, Takeuchi H. A study of tubo-ovarian and ovarian abscesses, with a focus on cases with endometrioma. J Obstet Gynaecol Res 1997;23:421-6.

50. Busacca M,Chiaffarino F, Candiani M, Vignali M, Bertulessi C, Oggioni G, et al. Determinants of longterm clinically detected recurrence rates of deep, ovarian and pelvic endometriosis. Am J Obstet Gynecol 2006;195:426-32.

51. Kikuchi I, Takeuchi H, Kitade M, Shimanuki H, Kumakiri J, Kinoshita K. Recurrence rate of endometriomas following a laparoscopic cystectomy. Acta Obstet Gynecol Scand 2006;85:1120-4. 